\title{
Long distance transmission through distributed erbium-doped fibers
}

\author{
Rottwitt, Karsten; Povlsen, Jørn Hedegaard; Bjarklev, Anders Overgaard
}

Published in:

Journal of Lightwave Technology

Link to article, DOI:

$10.1109 / 50.257977$

Publication date:

1993

Document Version

Publisher's PDF, also known as Version of record

Link back to DTU Orbit

Citation (APA):

Rottwitt, K., Povlsen, J. H., \& Bjarklev, A. O. (1993). Long distance transmission through distributed erbiumdoped fibers. Journal of Lightwave Technology, 11(12), 2105-2115. https://doi.org/10.1109/50.257977

\section{General rights}

Copyright and moral rights for the publications made accessible in the public portal are retained by the authors and/or other copyright owners and it is a condition of accessing publications that users recognise and abide by the legal requirements associated with these rights.

- Users may download and print one copy of any publication from the public portal for the purpose of private study or research.

- You may not further distribute the material or use it for any profit-making activity or commercial gain

- You may freely distribute the URL identifying the publication in the public portal

If you believe that this document breaches copyright please contact us providing details, and we will remove access to the work immediately and investigate your claim. 


\title{
Long Distance Transmission through Distributed Erbium-Doped Fibers
}

\author{
Karsten Rottwitt, Jørn Hedegaard Povlsen, and Anders Bjarklev
}

\begin{abstract}
High bit rate, all-optical long-distance transmission could be created through the combined use of loss-compensating gain in erbium-doped fibers and solitions. A detailed analysis of the distributed erbium-doped fiber, including the spectral-gain dependency, is combined with an optimum design of the transmission fiber and general bit-error-rate calculations. Changes in wavenumber, group velocity, and fiber dispersion due to erbium doping in a single-mode fiber are evaluated, and a reduction in bit-error rates due to the erbium spectral-gain profile is shown. Transmission through distributed erbium-doped fiber with 100 km separation between each pump-power station is shown, with a total bit-rate distance product of $55 \mathrm{~Gb} / \mathrm{s} \cdot \mathrm{Mm}$.
\end{abstract}

\section{INTRODUCTION}

$\mathrm{T}$ HERE ARE two types of long-distance soliton transmission methods. One is the lumped-amplifier technique, which has been analyzed in detail both theoretically (see, for example, [1]) and experimentally (see, for example, [2]). The other method is the distributed-amplifier technique, which can compensate for the fiber loss along the transmission line [3]. In all-optical soliton transmission using lumped amplification, the spacing between two amplifiers is limited to approximately $30 \mathrm{~km}$ due to bit-error-rate penalties resulting from excursion of the signal power [4]. The ideal distributed amplifier has no power excursions. However, due to the use of a finite pump power, instrinsic loss, fiber design, and spacing between each pump-power station, signal power excursions will exist. Distributed amplification may be achieved by use of the Raman effect [3] or rare-earth-doped fibers [5]. The latter method utilizes distributed erbium-doped fibers (d-EDFs), in which the whole transmission fiber is erbium-doped.

The principal object of this work is to examine the conditions for transmission of solitons through d-EDF having $100 \mathrm{~km}$ between each pump-power station. The design of a transparent d-EDF with $100-\mathrm{km}$ separation between each pump-power station was previously reported [6]. Here we focus on a detailed analysis of propagation properties of a d-EDF, in terms of bit-error rates (BER) including the spectral-gain profile.

The investigations reported herein are all based on a transmission system thousand of kilometers in overall

Manuscript received December 7, 1992; revised July 29, 1993. Work supported by the Danish Technical Research Council and by the $\mathrm{Na}$ tional Agency of Industry and Trade, Denmark.

The authors are with the Center for Broadband Telecommunications, Electromagnetics Institute, Technical University of Denmark, DK-2800 Lyngby, Denmark.

IEEE Log Number 9212989. length, which will contain many pump-power stations, all equidistantly spaced. Thus the signal energy exhibits a periodic perturbation. Faithful transmission is not automatically guaranteed in a periodically perturbed system. However, if the perturbation length, here the distance $L$ between two-pump-power stations, is less than eight times the soliton period, pulse distortion due to the perturbations may be neglected [3].

The perturbations that might conceivably lead to pulse distortion, even without considering noise accumulation, include not only the above-described effect of periodically varying gain, but also disturbances such as varying fiber cross sections and dispersion [7].

By assuming that the soliton period is larger than $L / 8$, the above subject of pulse stability will not be considered further in this work.

A major difference between lumped and distributed amplification is, as mentioned, the energy excursions. In a lumped-gain system, the path average signal power is different from the power after each amplifier and the launched-signal power. Thus the launched-signal power has to be adjusted to give a path average power equal to the soliton power [7], [8]. Further, a gain-dependent BER penalty is demonstrated, showing that the individual gain of each amplifier must be kept below $10 \mathrm{~dB}$ [4]. In an all-optical transmission system based on distributed amplification, the launched-signal power is identical to the path average power if the d-EDF is pumped bidirectionally and the erbium concentration and fiber refractiveindex profile is optimized [9]. In a 100-km-long d-EDF the energy excursions may be kept below $\pm 2 \mathrm{~dB}$. Generally, as long as the change in signal energy over an infinitesimal fiber piece is smaller in the d-EDF compared to the exponential energy decay in a lumped-gain transmission link, the d-EDF is preferable.

As previously mentioned, the signal-power excursions are very essential in nonlinear transmission. Thus it is important to use an accurate numerical model, including not only a complete description of the population inversion within the erbium, but also a true mode field description. In this work we have used the numerical model presented in [10].

\section{DESIGN OF d-EDF}

In an all-optical long-distance transmission line based on $\mathrm{d}-\mathrm{EDF}$, the fiber design requires greater concern than in the lumped-gain transmission line. In order to support solitary pulses, the mean dispersion has to be in the 
anomalous dispersion regime [1], [7]. This limits the choice of the numerical aperture (NA) and the $\mathrm{LP}_{11}$ cutoff wavelength $\lambda_{c}$ of the fiber. In general, a high NA fiber with $\lambda_{c}$ above $1 \mu \mathrm{m}$ is preferable [9].

Calculations of the noise performance comparing a step refractive-index profile and a triangular refractive-index profile show, in general, that there is a little advantage in using a step refractive-index profile considering generation of amplified spontaneous emission (ASE). On the other hand, a triangular refractive-index profile exhibits a lower additional background loss at higher NA values and a wider anomalous dispersion range. Considering other types of refractive-index profiles (i.e., segmented-core refractive-index profiles), the transmission properties are well approximated using a triangular refractive-index profile, and our results will be qualitatively valid also for such fiber designs. Therefore, we will focus on a triangular refractive-index profile.

In a long d-EDF the background loss is very essential due to both pump-power requirement and generation of ASE. The background loss is highly dependent on the fiber refractive-index profile, including the NA, and the use of dispersion-shifted fiber. Furthermore, the background loss is changed when considering alternative refractive-index-raising codopant materials. The background loss combined with the relative long length of fiber makes the $1480-\mathrm{nm}$ pumping band the only useful band. In short high-gain amplifiers, the influence on the background loss from the index-raising codopant material is unimportant. Thus, aluminum is considered as a very useful index-raising material. However, in the case of d-EDF the additional background loss introduced by aluminum is of great importance. Thus in this situation germanium is used as the index-raising material and aluminum is only considered for changing the emission and absorption cross-spectra of the d-EDF.

In [11] a fiber consisting of $90 \%$ germanium and $10 \%$ aluminum is considered. In this fiber, the index-raising material is germanium and the weak aluminum concentration is only sufficient to dominate the absorption and emission cross-section spectra. In [12] it is shown that if the additional background loss due to the use of aluminum is kept below $0.05 \mathrm{~dB} / \mathrm{km}$, the combined germanium/aluminum fiber is preferable. In the fiber design described in [11], where only $10 \%$ aluminum is added to a germanium-codoped fiber, the radius of the erbium profile is approximately identical to the fiber core radius. However, Simpson et al. [13] used a seed fiber technique to fabricate a d-EDF. In their fiber an aluminum-codoped erbium fiber is inserted into the fiber preform of a pure germanium single-mode fiber. This fiber, where the erbium is confined within the seed fiber, however, shows spectra dominated by germanium.

In general, the best noise performance is achieved using an erbium dopant radius as small as possible [14]. In the remaining text, we will concentrate on a pure germanium-codoped fiber with confined erbium, having an erbium radius 0.02 times the core radius and a background loss of $0.24 \mathrm{~dB} / \mathrm{km}$. Our results, however, are qualitatively independent on these parameters.

Also, the signal wavelength requires attention because the gain effective-signal wavelength for short high-gain amplifiers differs from the pump effective-signal wavelength giving transparency for the lowest pump-power requirements and the lowest noise figure. In short highgain amplifiers the most beneficial signal wavelength is mainly determined by the highest peak in the emission cross-section spectrum. The absorption is significant in long-length amplifiers pumped using a finite pump power at $1480 \mathrm{~nm}$ and the fraction of emission cross section to absorption cross section determines the signal wavelength. In [6] it is shown that 1.534 and $1.554 \mu \mathrm{m}$ are the two signal wavelengths of greatest importance. Both will be considered in the following text.

The erbium concentration is the final design parameter that we will consider. In Fig. 1 the necessary pump power for transparency as a function of the erbium concentration is shown in a germanium-codoped fiber for the two signal wavelengths 1.534 and $1.554 \mu \mathrm{m}$, respectively. Going toward a negligible erbium concentration, an increased pump power is needed to excite an increasingly higher percentage of erbium ions in order to cancel out the background loss. On the contrary, at very high erbium concentrations the increased absorption of the signal due to the erbium itself is cancelled out only by also increasing the pump power. This defines an optimum erbium concentration $\rho_{\text {opt }}$, where the pump power is minimized. Figure 1 clearly illustrates the optimum erbium concentration. At the erbium concentration denoted the critical concentration, $\rho_{c}$, the necessary pump power for transparency goes toward infinity. The value of $\rho_{c}$ is given by the background loss and the erbium cross-section spectra as

$$
\rho_{c}=\frac{\alpha_{I}}{\Gamma_{s}} \frac{\sigma_{e}^{p}+\sigma_{a}^{p}}{\sigma_{a}^{p} \sigma_{e}{ }^{s}-\sigma_{a}^{p} \sigma_{a}^{s}},
$$

where $\alpha_{l}$ is the intrinsic loss, $\sigma_{a}^{p}$ and $\sigma_{e}^{p}$ are the absorption and emission cross sections at the pump wavelength, and $\sigma_{a}^{s}$ and $\sigma_{e}^{s}$ are the absorption and emission cross sections at the signal wavelength. $\Gamma_{s}$ is the erbium confinement factor given by

$$
\Gamma_{s}=\frac{\int_{0}^{a_{\mathrm{dot}}} I r d r}{\int_{0}^{\infty} I r d r},
$$

where $a_{\mathrm{dot}}$ is the erbium dopant radius, $r$ is the radial coordinate in the fiber, and $I$ is the signal-mode intensity.

When the pump power is increased, the Raman effect has to be included in a realistic description [15], [16]. Including the Raman effect, the case of using an infinite pump power at the critical erbium concentration is translated into a high (near $400-\mathrm{mW}$ ), but finite, pump power when operating at an absent erbium concentration. Operating at the optimum concentration, the pump power is at a level where the Raman effect is negligible. 


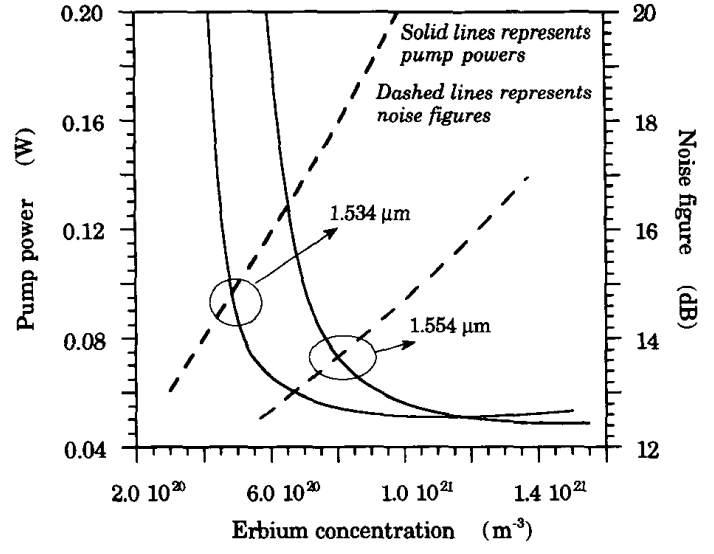

Fig. 1. The necessary pump power for transparency and the corresponding noise figure as a function of erbium concentration. The curves are calculated for two different values of the signal wavelength.

Because the noise figure, in general, increases for increasing erbium concentration [17], in agreement with Fig. 1 , the erbium concentration $\rho$ has to be in the range $\rho_{c}<\rho<\rho_{\text {opt }}$ and, including the Raman effect, the minimum noise figure is found near the critical erbium concentrantion [15].

\section{Pulse Propagation through d-EDF}

Describing pulse propagation through a d-EDF, the propagation constant $\beta$ includes contributions from the $\mathrm{GeSiO}_{2}$ host $\beta_{h}$ and the erbium dopant material $\beta_{d}$. The equation of wave propagation is written as

$$
\frac{\partial \Phi}{\partial z}=i\left(\beta_{h}+\beta_{d}\right) \Phi
$$

where $\Phi$ is the electrical field in the fiber and $z$ is the distance in the axial direction of the fiber. Expanding $\beta=\beta_{h}+\beta_{d}$ in a Taylor series around the optical angular carrier frequency, $\omega_{0}$, given by the signal wavelength, (1) is written in the form

$$
\begin{aligned}
\frac{\partial \Phi}{\partial z}=i\left(\beta_{0}+\beta_{1}(\omega\right. & \left.-\omega_{0}\right) \\
& \left.+\frac{1}{2} \beta_{2}\left(\omega-\omega_{0}\right)^{2}+\beta_{\mathrm{NL}}\right) \cdot \Phi,
\end{aligned}
$$

where $\beta_{0}=\beta\left(\omega=\omega_{0}\right)$ and $\beta_{1}$ and $\beta_{2}$ are the first- and second-order derivative of $\beta$ in $\omega=\omega_{0}$. Equation (2) includes the nonlinear Kerr effect through $\beta_{\mathrm{NL}}$. In general, each coefficient of $\beta$ in (2) is complex and consists of the two contributions $\beta_{h}$ and $\beta_{d}$. By taking the inverse Fourier transformation, $\omega$ is replaced by the differential operator $i(\partial / \partial t)$ and $\Phi(z, \omega)$ is replaced by $\psi(z, t)$, where $t$ denotes the time variable. Further, introducing an optical carrier wave $\exp \left(i\left(\operatorname{Re}\left[\beta_{0}\right] z-\omega_{0} t\right)\right)$ and a pulse envelope function $\varphi(z, t)$ through $\psi(z, t)=\varphi(z, t)$. $\exp \left(i\left(\operatorname{Re}\left[\beta_{0}\right] z-\omega_{0} t\right)\right)$, and finally changing to a re- tarded time frame by $t \rightarrow t-z / v_{g}$, where the group velocity $v_{g}=1 / \operatorname{Re}\left[\beta_{1}\right],(2)$ is rewritten in the form

$$
\begin{aligned}
\varphi_{z}+\frac{1}{2} i \operatorname{Re}\left[\beta_{2}\right] \varphi_{t t} & -i \beta_{\mathrm{NL}} \varphi \\
& =-\operatorname{Im}\left[\beta_{0}\right] \varphi+\frac{1}{2} \operatorname{Im}\left[\beta_{2}\right] \varphi_{t t},
\end{aligned}
$$

where it is assumed that $\operatorname{Im}\left[\beta_{1}\right]=0 . \operatorname{Re}\left[\beta_{2}\right]$ is related to the fiber dispersion $D$ through $D=-\left(2 \pi c / \lambda_{s}^{2}\right)$. $\operatorname{Re}\left[\beta_{2}\right]$, where $\lambda_{s}$ is the signal wavelength and $c$ is the velocity of light.

With the above definition of the propagation constant,

$$
\beta_{0}=\operatorname{Re}\left[\beta_{0 h}+\beta_{0 d}\right]+i \operatorname{Im}\left[\beta_{0 h}+\beta_{0 d}\right] .
$$

$\operatorname{Im}\left[\beta_{0 h}\right]$ is the intrinsic loss in the glass host fiber and $\operatorname{Im}\left[\beta_{0 d}\right]$ is the gain or attentuation introduced by the dopant material. In the ideal distributed fiber, these terms counterblanace each other at every point $z$, that is,

$$
\exp \left(-\operatorname{Im}\left[\beta_{0 h}+\beta_{0 d}\right] \cdot z\right)=1 .
$$

However, in a real d-EDF, $\operatorname{Im}\left[\beta_{0 h}(z)+\beta_{0 d}(z)\right]$ defines the relative gain through $g(z)=\operatorname{Im}\left[\beta_{0 h}(z)+\beta_{0 d}(z)\right]$, and in the transparent d-EDF,

$$
\exp \left(-\int_{0}^{L} g(z) d z\right)=1,
$$

where $L$ is the length of the d-EDF. It must be noted that if the gain curvature introduces additional losses, the gain resulting from the d-EDF has to be increased accordingly. We return to the gain curvature in the following text, but the spectral gain shape is calculated under the assumption of (6).

The spectral dependence of $\beta_{0 d}$ is displayed in Fig. 2, which shows the gain in a transparent germanium-codoped d-EDF with a signal wavelength at $1.534 \mu \mathrm{m}$. In this work we have assumed that the background loss $\exp \left(-\operatorname{Im}\left[\beta_{0 h}\right] \cdot L\right)$ equals $24 \mathrm{~dB}$ over the entire spectral range from 1.52 to $1.58 \mu \mathrm{m}$.

The real parts of $\beta_{0 h}$ and $\beta_{0 d}$ define the wavenumber of the carrier wave and are related to the imaginary parts of $\beta_{0 h}$ and $\beta_{0 d}$ through the Kramer-Kronig relation. The real part of $\beta_{0 d}$ may be calculated assuming that the optical power gain, $G=\exp \left(-\operatorname{Im}\left[\beta_{o d}\right] \cdot L\right)$, at the signal output end of the d-EDF has a Lorentzian spectral shape given by

$$
G=k \cdot \frac{1}{1+(\lambda / B)^{2}},
$$

where $\lambda$ is transformed to be centered around the signal wavelength $\lambda_{s}$, that is, $\lambda=0$ is equivalent to the signal wavelength. From Fig. $2, \lambda_{s}=1.534 \mu \mathrm{m}, k=24.0 \mathrm{~dB}$, and $B=3.1 \mathrm{~nm}$. To investigate an eventual influence from the nearest peaks in the grain spectrum, (7) is expanded to

$$
G=k \cdot \frac{1}{1+1+(\lambda / B)^{2}}+k_{1} \cdot \frac{1}{1+\left(\left(\lambda-\lambda_{1}\right) / B_{1}\right)^{2}} .
$$




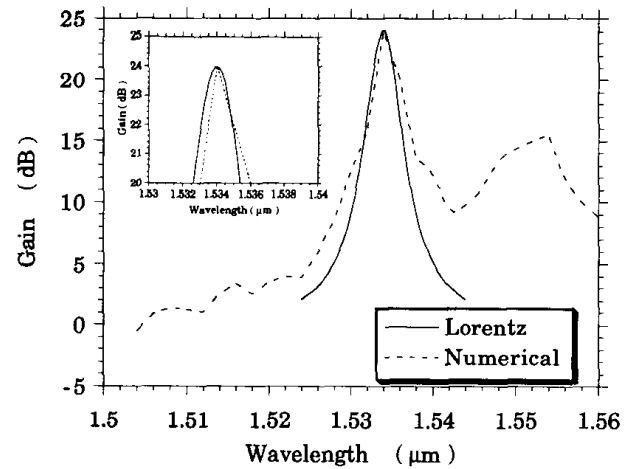

Fig. 2. The Lorentzian fit used in the calculations together with the numerical calculated gain spectrum. The inset figure demonstrates that the fit simply is made according to the gain peak wavelength and the 3-dB bandwidth.

From Fig. 2, the nearest peak to the gain around 1.534 $\mu \mathrm{m}$ is $\lambda_{1}=1.554 \mu \mathrm{m}$, with $k_{1}=15.6 \mathrm{~dB}$ and $B_{1}=8.3$ $\mathrm{nm}$. Using the Kramer-Kronig relation, we achieve

$$
\begin{aligned}
\operatorname{Re}\left[\beta_{d}(\lambda)\right]= & \left(k \cdot \frac{\lambda / B}{1+(\lambda / B)^{2}}\right. \\
& \left.+k_{1} \frac{\left(\lambda-\lambda_{1}\right) / B_{1}}{1+\left(\left(\lambda-\lambda_{1}\right) / B_{2}\right)^{2}}\right) \cdot \frac{1}{20 \log e} .
\end{aligned}
$$

In the following text we express $\left(\lambda-\lambda_{1}\right) / B_{1}$ as $X$. Due to the second gain peak around $1.554 \mu \mathrm{m}$, the carrier wavenumber is shifted $k_{1} X /\left(1+X^{2}\right)=0.65$ over the 100 -km-long d-EDF. For comparison, $\operatorname{Re}\left[\beta_{0 h}\right]$ approximates $5.9 \cdot 10^{6} \mathrm{~m}^{-1}$ at the signal wavelength. The shift in group velocity $\operatorname{Re}\left[\beta_{1 d}(\lambda=0)\right]$ is given by

$$
\begin{gathered}
\left.\frac{\partial}{\partial \omega} \operatorname{Re}\left[\beta_{d}(\lambda)\right]\right|_{\lambda=0}=\frac{\lambda_{s}^{2}}{2 \pi c} \cdot\left(\frac{k}{B}+\frac{k_{1}}{B_{1}} \cdot \frac{1-X^{2}}{\left(1+X^{2}\right)^{2}}\right) \\
\cdot \frac{1}{20 \log e} .
\end{gathered}
$$

With the previous values, a group velocity shift of $1.1 \mathrm{ps}$ over $100 \mathrm{~km}$ is found. For comparison, the group delay estimated in the host fiber is $4.8 \mathrm{~ns} / \mathrm{m}$. The contribution from the second gain peak is approximately $3 \%$, and the contribution decreases with increasing $X$. The derivative of $\operatorname{Im}\left[\beta_{0 d}(\lambda)\right]$ is the slope of the gain, which approximates zero. More accurately, the wavelength where the gain slope equals zero is

$$
\lambda_{s}=\frac{k_{1}}{k} \cdot\left(\frac{B}{B_{1}}\right)^{2} \frac{\lambda_{1}}{\left(1+\left(\lambda_{1} / B_{1}\right)^{2}\right)^{2}} .
$$

Insertion of fiber parameters gives $\lambda_{s} \approx \lambda_{1} \cdot 10^{-3}$. This wavelength is within the spectral width of the pulse. Thus we continue under the approximation that $\partial \operatorname{Im}\left[\beta_{0 d}(\omega)\right] / \partial \omega$ equals zero around the carrier frequency.
The second derivative of $\operatorname{Re}\left[\beta_{d}\right]$ with respect to the angular frequency $\omega$ results in a change in the dispersion. The change in dispersion is $\Delta D=-\left(2 \pi c / \lambda_{s}^{2}\right)$. $\operatorname{Re}\left[\beta_{2 d}(\lambda=0)\right]$. Here

$$
\begin{aligned}
\left.\frac{\partial^{2}}{\partial \omega^{2}} \operatorname{Re}\left[\beta_{d}(\lambda)\right]\right|_{\lambda=0}= & \frac{-\lambda_{s}^{2}}{2 \pi c} \cdot\left(\left.\frac{\lambda_{s}}{\pi c} \cdot \frac{\partial \operatorname{Re}\left[\beta_{d}(\lambda)\right]}{\partial \lambda}\right|_{\lambda=0}\right. \\
& \left.+\left.\frac{\lambda_{s}^{2}}{2 \pi c} \frac{\partial^{2} \operatorname{Re}\left[\beta_{d}(\lambda)\right]}{\partial \lambda^{2}}\right|_{\lambda=0}\right) \\
& \cdot \frac{1}{20 \log e} .
\end{aligned}
$$

By insertion in (12) we obtain a $\left(1.3 \cdot 10^{-3}\right)$-ps / nm change in dispersion achieved over 24-dB gain, here over $100 \mathrm{~km}$ of d-EDF. This contribution is negligible and we therefore conclude that the dispersion is unchanged by doping the fiber in order to cancel out the background loss.

The curvature of the imaginary part of $\beta_{d}$ is

$$
\begin{aligned}
& \left.\frac{\partial^{2} \operatorname{Im}\left[\beta_{d}(\lambda)\right]}{\partial \omega^{2}}\right|_{\lambda=0} \\
& \quad=\left\{\frac{\lambda_{s}^{3}}{(\pi c)^{2}} \frac{k_{1}}{B_{1}} \frac{X}{\left(1+X^{2}\right)^{2}}\right. \\
& \left.+\frac{2 \lambda_{s}^{4}}{(2 \pi c)^{2}}\left(\frac{k}{B^{2}}+\frac{k_{1}}{B_{1}^{2}} \frac{1-3 X^{2}}{\left(1+X^{2}\right)^{3}}\right)\right\} \frac{1}{20 \log e} .
\end{aligned}
$$

In Appendix A we show, on the basis of results from [9] and [18], that a positive curvature of the gain reduces the variance of the arrival time of the signal pulses $\left\langle\delta t^{2}\right\rangle$ in the timing jitter denoted in the next equation. The reduction is expressed through

$$
\left\langle\delta t^{2}\right\rangle=\left.\left\langle\delta t^{2}\right\rangle\right|_{\mathrm{GH}} \cdot \frac{3}{2} \cdot \frac{3+e^{2 \alpha z}+2 \alpha z-4 e^{\alpha z}}{(\alpha z)^{3}},
$$

where $\alpha=-\left(4 a^{2} / 3\right) \cdot\left(\frac{1}{2} \operatorname{Im}\left[\beta_{2 d}(\lambda=0)\right]\right)$ and $a=2$. $\ln (\sqrt{2}+1) / \tau .\left.\left\langle\delta t^{2}\right\rangle\right|_{\mathrm{GH}}$ is the timing jitter without considering the curvature of the d-EDF gain profile, known as the Gordon-Haus variance [4]. By insertion of the previous parameters, $\operatorname{Im}\left[\beta_{2 d}(\lambda=0)\right]=0.88(\mathrm{ps})^{2}$ at $\lambda_{s}$ $=1.534 \mu \mathrm{m}$. Figure 3 illustrates on a decibel scale the reduction in the variance of the arrival time of the signal pulses found using (14) as

$$
10 \cdot \log _{10}\left(\frac{3}{2} \frac{3+\exp (2 \alpha z)+2 \alpha z-4 \exp (\alpha z)}{(\alpha z)^{3}}\right)
$$

and the subsequent reduction in BER as a function of the pulsewidth, expressed through $10 \cdot\left(\log _{10}\left(\mathrm{BER}_{\mathrm{GH}}\right)-\right.$ $\log _{10}\left(\mathrm{BER}_{\mathrm{RGH}}\right)$ ), where $\mathrm{BER}_{\mathrm{GH}}$ and $\mathrm{BER}_{\mathrm{RGH}}$ are found using (22) (to be explained in the following text) in combination with $\left.\left\langle\delta t^{2}\right\rangle\right|_{\mathrm{GH}}$, respectively, $\left\langle\delta t^{2}\right\rangle$. The achieved reductions are showed after 6000 and $9000 \mathrm{~km}$. Figure 3 also shows the length giving a BER of $10^{-9}$, denoted as 


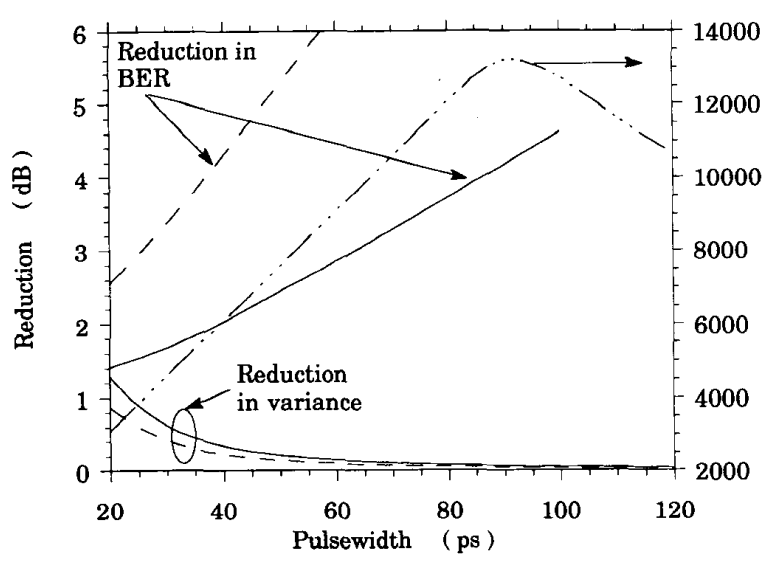

Fig. 3. The reduction in timing jitter variance and BER as a function of the pulsewidth. The error-free length is also shown as a function of the pulsewidth. Dashed curves represent $6000 \mathrm{~km}$, solid curves $9000 \mathrm{~km}$.

the error-free length (EFL). In the figure a fiber dispersion of $1.8 \mathrm{ps} /(\mathrm{nm} \cdot \mathrm{km})$ has been used and the BER was calculated as described in detail in the succeeding sections, having a 14-dB noise figure and assuming that the receiver is sensitive to 20 signal modes $M=20$. It is worth noting that the reduction in BER descreases with the distance of propagation even though the reduction in timing jitter increases. This is explained due to the strongly increasing timing jitter with increasing distance of propagation. Because the timing jitter increases more rapidly than the reduction factor (14), the reduction in BER decreases with the propagating distance. Figure 3 clearly illustrates that the influence from the spectral gain shape is highest at short pulsewidths. However, it must be noted that for decreased pulsewidth, the spectral pulsewidth increases and will consequently be destroyed due to the narrow bandwidth of the amplifier [19]. Furthermore, at small pulsewidths the curvature of the gain leads to the need for additional gain, as mentioned earlier, which may result in inhibited noise performance [20], [21].

In summary, the gain profile created through the erbium doping results in a vanishing change in both the carrier wavenumber, the group velocity $v_{g}$, and the fiber dispersion. However, the gain profile leads to a reduction in timing jitter due to the curvature of the gain profile around the signal wavelength. This conclusion is based on the gain spectrum calculated on the basis of the emission and absorption cross-section spectra of erbium in the range between 500 and $1700 \mathrm{~nm}$.

\section{Noise Accumulation}

In nonlinear long-distance transmission, where the nonlinear Kerr effect and the spectral dispersion counterbalance each other in generation of solitary pulses, there are two major error sources. One is the jitter in the arrival times of the signal pulses, and the other is amplitude noise, which degenerates the signal-to-noise ratio at the detector.
Each amplifier in a chain generates amplified spontaneous emission (ASE). The ASE induces a random shift in the carrier frequency of the soliton which interacts with the fiber dispersion to produce a random timing jitter of the arrival time of the signal pulses. Additionally, the system must be designed to achieve an acceptable signalto-noise ratio at the detector, where the dominant noise effect is to be the beating between the optical signal and the ASE (signal spontaneous beat noise).

In the previous section an expression for the variance of the arrival time of the signal pulses, denoted as the timing jitter in the following, was given in (14). The signal-to-noise ratio for a mark at the detector end is given by

$$
\frac{S}{N}=\frac{E_{s}+2 M \tilde{E}}{2 \tilde{E}}=\frac{1}{2} \cdot \frac{\langle E\rangle \cdot 2}{R_{g} \alpha_{\mathrm{ASE}} \cdot L_{\mathrm{tot}} \cdot h \gamma_{s}}+M,
$$

where $E_{s}$ is the signal energy after each amplifier, $\tilde{E}$ is the noise energy after each amplifier, and $M$ is the number of signal modes to which the receiver is sensitive [9]. $R_{g}$ is the path average integral $R_{g}=(1 / L) \cdot \int_{a}^{L} e^{g(z)} d z$, where $g(z)$ is defined in (6). $\alpha_{\mathrm{ASE}^{1 / 2}}$ is the number of generated ASE photons per meter in the signal mode. Finally, $L_{\text {tot }}$ is the total transmission length and $h \nu_{s}$ is the photon energy at the optical signal frequency. In both the timing jitter and the signal-to-noise ratio, the factor $R_{g}$. $\alpha_{\mathrm{ASE}}$ is essential.

In the case of lumped amplification, $R_{g} \cdot \alpha_{\mathrm{ASE}}$ may be expressed in a more simple form as

$$
\begin{aligned}
\alpha_{\mathrm{ASE}} \cdot R_{g} & =\frac{F G-1}{L} \cdot \frac{1}{L} \cdot \int_{0}^{L} e^{-\alpha z} d z \\
& =\frac{F G-1}{L} \cdot \frac{G-1}{G \ln G},
\end{aligned}
$$

where $\alpha$ is the attenuation constant in each passive fiber section, $L$ is the distance between each amplifier, $F$ is the noise figure, and $G$ is the gain of each amplifier. Assuming that each amplifier is pumped at $980 \mathrm{~nm}$, the noise figure $F=2-1 / G$, and simply

$$
\alpha_{\mathrm{ASE}} R_{g}=2 \alpha \cdot\left(\frac{G-1}{\ln G}\right)^{2} \frac{1}{G} .
$$

In a d-EDF having an erbium concentration equal to the critical erbium concentration and an infinite pump power at $1480 \mathrm{~nm}$, the highest possible population inversion in the erbium is achieved along the complete transmission line and $R_{g}=1$. In Appendix B the noise figure in this situation is shown to be $F=2 \cdot q \cdot \ln \left(D_{B}\right)+1$, where $D_{B}$ is the total background loss within the fiber and

$$
q=\frac{\sigma_{e}^{s} \sigma_{a}^{p}}{\sigma_{e}^{s} \sigma_{a}^{p}-\sigma_{a}^{s} \sigma_{e}^{p}} .
$$

Having $D_{B}=24 \mathrm{~dB}$ and pumping at $1480 \mathrm{~nm}, q=1.73$ $\left(\lambda_{s}=1.534 \mu \mathrm{m}\right)$, which gives $F=13.0 \mathrm{~dB}$, whereas $q=$ $1.47\left(\lambda_{s}=1.554 \mu \mathrm{m}\right)$ and, accordingly, $F=12.4 \mathrm{~dB}$. These 
values are also seen as the asymptotic values in Fig. 1. Thus in the d-EDF, $\alpha_{\mathrm{ASE}} R_{g}=(F-1) / L \cdot 1$ in the ideal case, and consequently,

$$
\alpha_{\mathrm{ASE}} R_{g}=2 \cdot q \cdot \alpha
$$

Calculating $R_{g} \cdot \alpha_{\mathrm{ASE}}$ in the lumped-gain transmission line, respectively, the d-EDF transmission line, using (17) and (19) it is evident that there exists a gain $G$ in the lumped-gain transmission line where $\alpha_{\mathrm{ASE}} R_{g}$ in the two situations are identical.

To prove that an improved noise performance is achievable by changing from lumped to distributed amplification, we compare in Fig. $4 R_{g} \cdot \alpha_{\text {ASE }}$ for the two situations. Concerning lumped gain, $R_{g} \cdot \alpha_{\text {ASE }}$ is calculated under ideal conditions using (17), whereas $R_{g} \cdot \alpha_{\mathrm{ASE}}$ is calculated numerically assuming realistic input parameters for the d-EDF. In Fig. 4 the two curves displaying $R_{\mathrm{g}} \cdot \alpha_{\text {ASE }}$ cross for a length of $40 \mathrm{~km}$ equal to $10 \mathrm{-dB}$ gain in the case of lumped gain. For larger lengths between two amplifiers in the lumped-gain transmission line, $R_{g}$. $\alpha_{\mathrm{ASE}}$ is larger than in a d-EDF of similar length. Thus we conclude that for larger fiber spans, here exceeding 40 $\mathrm{km}$, in a lumped-gain transmission system, the penalty due to energy excursions will exceed the penalty in a d-EDF due to the incomplete inversion when pumping at 1480 $\mathrm{nm}$, and an improved noise performance is achievable using d-EDF.

Until now we have mainly focused on the noise figure expressing the noise performance around the signal wavelength. However, ASE power is emitted throughout the complete amplifier bandwidth. According to the spectral gain of the d-EDF shown in Fig. 2, the two signal wavelengths that need the highest concern are $1.534 \mu \mathrm{m}$, respectively, $1.554 \mu \mathrm{m}$. In general, ASE accumulation follows the expression

$$
\begin{aligned}
P_{\mathrm{ASE}}(z=N \cdot L) & =P_{\mathrm{ASE}}(Z=L) \cdot \sum_{n=1}^{N} G^{n-1} \\
& =P_{\mathrm{ASE}}(Z=L) \cdot \frac{G^{N}-1}{G-1},
\end{aligned}
$$

where $G$ is the gain within the bandwidth giving $P_{\mathrm{ASE}}$. In Figs. 2 and 5 the gain profile is shown for a d-EDF optimized for the signal wavelengths 1.534 and $1.554 \mu \mathrm{m}$, respectively. A triangular refractive-index profile with a core radius of $3.5 \mu \mathrm{m}$ giving the $\mathrm{LP}_{11}$ cutoff wavelength $\lambda_{c}=1.036 \mu \mathrm{m}$ and an index difference of $13.75 \cdot 10^{-3}$ equal to a numerical aperture of 0.2 has been used. These parameters result in a fiber dispersion of $1.8 \mathrm{ps} /(\mathrm{nm} \cdot \mathrm{km})$ and an effective core area $A_{\text {eff }}$ of $41 \mu \mathrm{m}^{2}$. Erbium is introduced in the core with an erbium radius 0.02 times the core radius. The $100-\mathrm{km}$ fiber is pumped equally from both ends with $1480-\mathrm{nm}$ light, and a total pump power of $100 \mathrm{~mW}$ is used. The intrinsic loss is for this germaniumcodoped d-EDF assumed to be $0.24 \mathrm{~dB} / \mathrm{km}$. The erbium concentration is adjusted to give $0-\mathrm{dB}$ gain, the actual values of the noise figure, the global ASE power, the

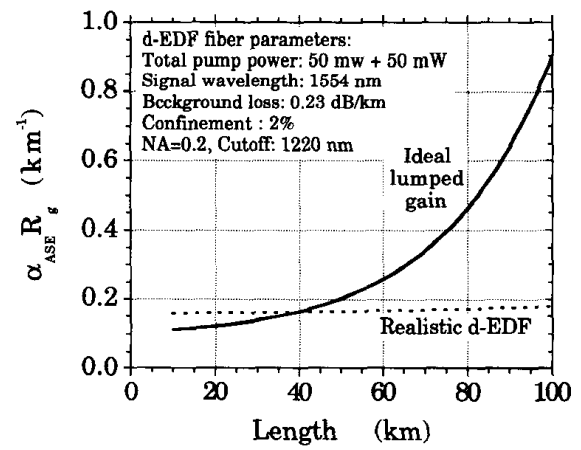

Fig. 4. The $\alpha_{\mathrm{ASE}} \cdot R_{g}$ product as a function of the length between two pump power stations in lumped-gain assuming a background loss of 0.25 $\mathrm{dB} / \mathrm{km}$.

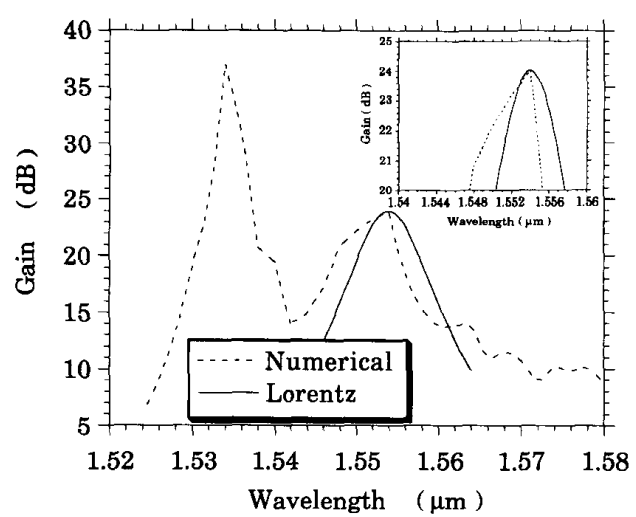

Fig. 5. The numerical calculated gain spectrum for the $100-\mathrm{km}-$ long d-EDF with transparency at the $1.554-\mu \mathrm{m}$ signal wavelength and the Lorentzian fit. The inset spectrum is a close-up view of the gain spectrum around the signal wavelength.

erbium concentration $\rho$, the critical erbium concentration, $\rho_{c}$, and the gain variation are shown in Table I. All values are based on a $0.1-\mathrm{mW}$ signal power. From the table it is obvious that $\lambda_{s}=1.554 \mu \mathrm{m}$ gives the lowest noise figure, even though $P_{\mathrm{ASE}}$ is highest in this fiber. In both fibers the ASE power is concentrated around 1.534 $\mu \mathrm{m}$, due to the profile of the emission cross-section spectra of the fiber. This explains the relation between $F$ and $P_{\mathrm{ASE}}$ in the two situations. At $\lambda_{s}=1.554 \mu \mathrm{m}, 10-\mathrm{dB}$ gain is accomplished around $1.534 \mu \mathrm{m}$. Within a bandwidth of $2 \mathrm{~nm}$ an ASE power of $3.8 \mu \mathrm{W}$ is found. Without any further filtering a maximum of three d-EDF may be cascaded if the signal power has to exceed the ASE power.

If instead, the signal wavelength is changed to 1.534 $\mu \mathrm{m}$, ASE power at any other wavelength is attenuated. Around the $1.554-\mu \mathrm{m}$ peak in the gain spectrum an ASE power of $0.23 \mu \mathrm{W}$ is emitted from one amplifier section within a $2-\mathrm{nm}$ bandwidth. After an infinite number of cascaded amplifiers this ASE power is increased to only $0.27 \mu \mathrm{W}$, which is essentially lower than the signal power. 
TABLE I

Characteristic Figures for a D-EDF USED AT 1.534 AND $1.554 \mu \mathrm{M}$

\begin{tabular}{lcl}
\multicolumn{3}{c}{ AT 1.534 AND $1.554 \mu \mathrm{M}$} \\
\hline & $\lambda_{s}=1.534 \mu \mathrm{m}$ & $\lambda_{s}=1.554 \mu \mathrm{m}$ \\
\hline$F(\mathrm{~dB})$ & 14.53 & 13.26 \\
$P_{\mathrm{ASE}}(\mu \mathrm{W})$ & 5.2 & 17.8 \\
$\rho\left(\mathrm{m}^{-3}\right)$ & $2.80 \cdot 10^{23}$ & $4.45 \cdot 10^{23}$ \\
$\rho_{c}\left(\mathrm{~m}^{-3}\right)$ & $1.84 \cdot 10^{23}$ & $3.30 \cdot 10^{23}$ \\
$\Delta G(\mathrm{~dB})$ & $+1.38 \rightarrow-1.52$ & $+1.02 \rightarrow-1.07$ \\
\hline
\end{tabular}

From this it is evident that additional filters have to be included if the signal wavelength $1.554 \mu \mathrm{m}$ is used. On the contrary, the signal wavelength $1.554 \mu \mathrm{m}$ exhibits an improved noise performance, therefore an increased transmission length may be achieved, and the use of discrete filters will reduce both the timing jitter and the backward and forward propagating ASE.

\section{BER IN d-EDF SYSTEMS}

The fundamental goal in the design of an all-optical digital transmisstion line is to minimize the amount of optical noise power accompanying the signal in order to achieve a given BER. In nonlinear transmission, where the pulse broadening is minimized with use of optical solitary pulses, there are (as mentioned earlier) two major error sources denoted timing jitter and amplitude noise or noise power error.

From [9] the variance of the arrival time of the pulses is known to be

$$
\left.\left\langle\delta t^{2}\right\rangle\right|_{\mathrm{GH}}=\frac{2 \cdot \ln (\sqrt{2}+1)}{9} h \frac{D L_{\mathrm{tot}}^{3} \cdot n_{\mathrm{ke}}}{\tau A_{\mathrm{eff}}} \cdot \frac{\alpha_{\mathrm{ASE}}}{2} R_{g},
$$

where $n_{\mathrm{ke}}$ is the nonlinear Kerr coefficient and the other parameters are as defined previously.

Due to the spectral gain shape, a reduction in the Gordon-Haus variance is achieved and the true variance is expressed in (14). This variance may be translated to bit-error rates assuming that the arrival time of the pulses follows a Gaussian distribution. Assuming further that a pulse arriving within the time slot $2 t_{w}$ is detected correctly and a pulse arriving outside this time slot results in an error, the BER is expressed as

$$
\mathrm{BER}_{\mathrm{TJ}}=\operatorname{erfc} \frac{t_{w}}{\sqrt{2} \sigma}
$$

where erfc is the error function and

$$
\sigma=\sqrt{\left\langle\delta t^{2}\right\rangle}
$$

Combining the electrical signal-to-noise ratio with the receiver model and BER calculations of [22], BER from amplitude noise is found in [9] and is expressed in terms of $R_{g} \cdot \alpha_{\mathrm{ASE}}$ as

$$
\mathrm{BER}_{\mathrm{NP}}=\frac{1}{2} \operatorname{erfc} \frac{Q}{\sqrt{2}},
$$

where

$$
Q=\frac{S_{1}}{\sqrt{2 S_{1}}+M}, \quad S_{1}=\frac{\langle E\rangle \cdot 2}{R_{g} \cdot \alpha_{\mathrm{ASE}} \cdot h \nu_{s} \cdot L_{\mathrm{tot}}} .
$$

The former sections have mainly been concentrated on minimization of the noise figure. For a transmission line, the dispersion, the effective core area, and the pulsewidth are also design parameters. However, there is a strong relation between the fiber dispersion and the effective core area. By having a high fixed relative numerical aperture fiber (NA > 0.15), the dispersion may be reduced without significant change in either the effective core area or $R_{g} \cdot \alpha_{\mathrm{ASE}}$ [9]. Thus by changing the cutoff wavelength slightly by changing the fiber core radius, the fiber dispersion may be changed. Also the pulsewidth may be adjusted slightly. By a reduction in $\tau$, the spectral pulsewidth is increased and the soliton period is decreased. At the $1.534-\mu \mathrm{m}$ signal wavelength the spectral gain after $100 \mathrm{~km}$ of fiber shows the narrowest bandwidth of approximately $2 \mathrm{~nm}$. In the remaining text we will concentrate on pulsewidths larger than $10 \mathrm{ps}$, equivalent to a spectral pulsewidth less than $0.2 \mathrm{~nm}$.

According to the earlier demand $L / z_{0} \ll 8$, we find the restriction

$$
\tau^{2} \gg \frac{L}{8} \frac{\lambda^{2}}{\pi^{2} c} \cdot \frac{D}{0.332},
$$

where $L$ is the length of the d-EDF, $D$ is the fiber dispersion, and the other parameters are as defined earlier. Having a maximum signal wavelength of $1.554 \mu \mathrm{m}$ and a maximum dispersion $D=2.5 \mathrm{ps} /(\mathrm{nm} \cdot \mathrm{km})$, the pulsewidth has to exceed $10 \mathrm{ps}$.

Changing the pulsewidth $\tau$ also changes the mean signal power. However, looking at the required pump power for transparency and the noise performance at the optimum erbium concentration, these are almost independent of the signal power as shown in Fig. 6. Thus using erbium concentrations within the critical and the optimum concentration, $\tau$ may be changed within one or two decades without changing the noise performance and the required pump-power level.

According to [23] the final BER is a result of both timing jitter and noise power error. Equation (22) shows that the BER from timing jitter increases with an increase in the fraction

$$
\frac{\sqrt{\left\langle\delta t^{2}\right\rangle}}{t_{w}} \propto \frac{1}{\tau} \cdot \sqrt{\frac{D}{\tau}}
$$

whereas (24) shows that the BER from amplitude noise increases with an increase in the fraction

$$
\frac{\sqrt{2 S_{1}}+M}{S_{1}} \propto \sqrt{\frac{\tau}{D}} .
$$

Thus it follows that there is an optimum pulsewidth that gives a maximum transmission length for a fixed dispersion. In Fig. 7 the maximum transmission length giving a BER of $10^{-9}$ is illustrated as a function of the pulsewidth 


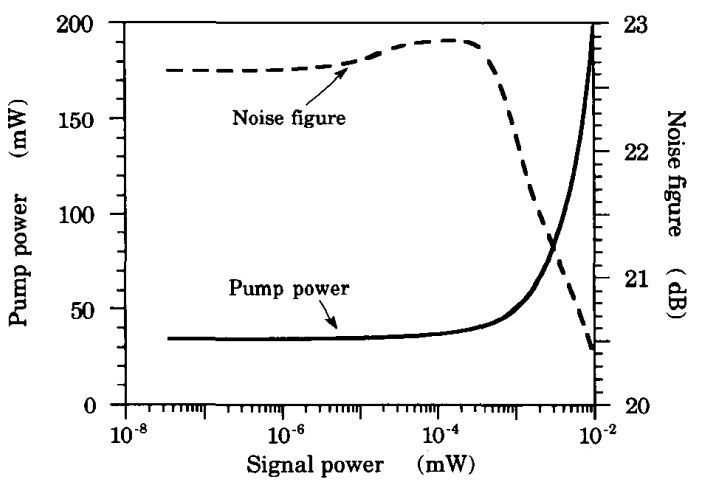

Fig. 6. The mean signal power dependency on the pump power and noise figure of a 100 -km-long d-EDF with an optimum erbium concentration.

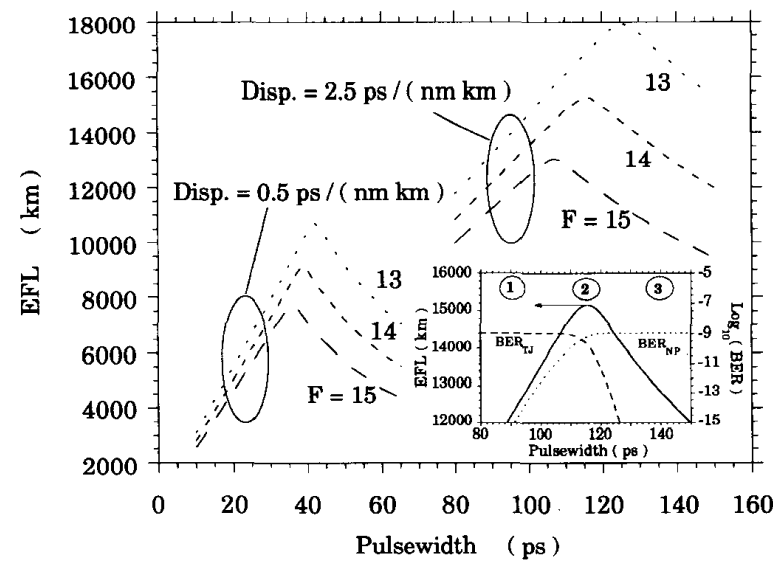

Fig. 7. The error-free length as a function of the pulsewidth. The EFL is shown for two values of fiber dispersion and three values of noise figure. The inset figure displays the BER curves resulting only from timing jitter and amplitude noise, having a noise figure of $14 \mathrm{~dB}$ and a fiber dispersion of $2.5 \mathrm{ps} /(\mathrm{nm} \cdot \mathrm{km})$.

for two values of the fiber dispersion and for each fiber dispersion, three values of the d-EDF noise figure $F$. The BERs resulting from timing jitter are calculated assuming that $2 t_{w}$ equals the time slot given by the bit rate. Furthermore, it is assumed that the detector is sensitive to $M=20$ signal modes. These choices do not qualitatively influence our results. The inset figure is a close-up view of the situation with a fiber dispersion of $2.5 \mathrm{ps} /(\mathrm{nm} \cdot \mathrm{km})$ and a noise figure of $14 \mathrm{~dB}$. The inset figure clearly displays the optimum $\tau$ in region 2 where both BER contributions are comparable. In region 1 for $\tau$ lower than the optimum, the major errors occur due to the jitter of the arrival time of the signal pulses, whereas it is errors due to the increased noise power relative to the signal power that are responsible for the limits in system length in region 3 . Even more interesting is the situation comparing the maximum transmission length at two different fiber dispersions. For increased fiber dispersion the maxi- mum system length may be increased using a lower bit rate. Having a fiber dispersion of $0.5 \mathrm{ps} /(\mathrm{nm} \cdot \mathrm{km})$ and a 14-dB noise figure, the maximum system length is 9000 $\mathrm{km}$ at a bit rate of $5 \mathrm{~Gb} / \mathrm{s}$ equivalent to a bit-rate distance product of $45 \mathrm{~Gb} / \mathrm{s} \cdot \mathrm{Mm}$. Reducing the noise Fig. by $1 \mathrm{~dB}$ leads to $10-\mathrm{Gb} / \mathrm{s} \cdot \mathrm{Mm}$ increase in the bit-rate distance product. Increasing the fiber dispersion to $2.5 \mathrm{ps} /(\mathrm{nm} \cdot \mathrm{km})$ for the $14-\mathrm{dB}$ noise figure increases the maximum system length to $15000 \mathrm{~km}$, but at a bit rate of $1.8 \mathrm{~Gb} / \mathrm{s}$, equivalent to a bit-rate distance product of $27 \mathrm{~Gb} / \mathrm{s} \cdot \mathrm{Mm}$. Looking at a fixed fiber dispersion the bit-rate distance product may increase in region 1 , whereas it will always decrease in region 3 . Thus optimum operation is always based on operation where errors due to jitter of the arrival time of the pulses exceed errors due to an increased noise power.

\section{Conclusion}

An optimum design for a distributed erbium-doped optical fiber is demonstrated. For a total pump power of $100 \mathrm{~mW}$ and $100-\mathrm{km}$ separation between two pump-power stations, a noise performance comparable to the best obtainable in a lumped-gain system, having approximately three amplifiers with $100 \mathrm{~km}$, is found. A detailed theoretical analysis of pulse propagation through distributed erbium-doped fiber is given. It is shown that the spectral-gain profile does not result in any significant change in the carrier wavenumber, the group velocity, or the fiber dispersion. However, the spectral-gain profile leads to a reduction in bit-error rates due to timing jitter of the arrival times of the signal pulses. Reductions of up to 4 $\mathrm{dB}$ are found in transmission lines of $9000-\mathrm{km}$ length.

Two signal wavelengths are of significant interest: 1.534 and $1.554 \mu \mathrm{m}$. Both are usable for transmission over several thousands of kilometers. However, use of 1.554 $\mu \mathrm{m}$ will need insertion of additional components in order to reduce propagation of amplified spontaneous emission. If insertion of such components is possible without increasing the noise figure more than $1 \mathrm{~dB}, 1.554 \mu \mathrm{m}$ is the optimum signal wavelength.

In transmission lines based on distributed amplification the pulsewidth and the fiber dispersion are two important design parameters. An optimum relation between pulsewidth and fiber dispersion is illustrated, and it is shown that optimum system operation will always be limited by timing jitter. A bit rate-distance product of 55 $\mathrm{Gb} / \mathrm{s} \cdot \mathrm{Mm}$ is illustrated.

\section{APPENDIX A \\ DERIVATION OF THE INFLUENCE FROM THE GAIN CURVATURE OF THE d-EDF}

The equation governing pulse propagation in a d-EDF is written in the form

$$
\begin{aligned}
\frac{\partial \varphi}{\partial z}+i \frac{\operatorname{Re}\left[\beta_{2}\right]}{2} \frac{\partial^{2} \varphi}{\partial t^{2}} & -i \gamma|\varphi|^{2} \varphi \\
& =-\Gamma(z) \varphi+\frac{\operatorname{Im}\left[\beta_{2}\right]}{2} \frac{\partial^{2} \varphi}{\partial t^{2}}
\end{aligned}
$$


where $\varphi$ is an envelope pulse of the optical carrier wave $\exp \left(i\left(\operatorname{Re}\left[\beta_{0}\right] z-\omega_{0} t\right)\right)$, propagating in the retarded time frame given by $t \rightarrow t-z / v_{g} . \beta_{2}$ is the second-order derivative of the propagation constant and the imaginary part is related to the gain profile introduced by the erbium as described in the main text, $\gamma$ includes the nonlinear Kerr effect, and $\Gamma(z)$ is the relative gain related to $g(z)$ in the main text through $\Gamma(z)=-g(z)$. For convenience, we use the notation $\epsilon=-\operatorname{Im}\left[\beta_{2}\right] / 2$ in the following analysis. Based on (A.1), the relations of energy and momentum conservation are expressed as

$$
\begin{gathered}
\frac{\partial}{\partial z} \int_{-\infty}^{\infty} \varphi^{*} \varphi d t=2 g(z) \cdot \int_{-\infty}^{\infty}|\varphi|^{2} d t+2 \epsilon \int_{-\infty}^{\infty}\left|\frac{\partial \varphi}{\partial t}\right|^{2} d t \\
\frac{\partial}{\partial z} \int_{-\infty}^{\infty}\left(\varphi^{*} \frac{\partial \varphi}{\partial t}-\varphi \frac{\partial \varphi^{*}}{\partial t}\right) d t \\
=2 g(z) \int_{-\infty}^{\infty}\left(\varphi^{*} \frac{\partial \varphi}{\partial t}-\varphi \frac{\partial \varphi^{*}}{\partial t}\right) d t \\
+2 \epsilon \int_{-\infty}^{\infty}\left(\frac{\partial \varphi^{*}}{\partial t} \frac{\partial^{2} \varphi}{\partial t^{2}}-\frac{\partial \varphi}{\partial t} \frac{\partial^{2} \varphi^{*}}{\partial t^{2}}\right) d t . \quad \text { (A.2b) }
\end{gathered}
$$

Assuming a solitary pulse is the solution to (A.1), where the radial electrical field dependency is included in $\gamma$,

$$
\varphi=A \cdot \operatorname{sech}(a \cdot t) e^{i \xi z} \cdot e^{i \omega t},
$$

where $\xi$ and $\omega$ are functions of $z$. $\xi$ represents a change in the carrier wavenumber, whereas $\omega$ is a change in the optical angular frequency $\omega_{0}$.

Insertion of $\varphi$ in (A.2a) and (A.2b) using $A=\eta \cdot a$ gives

$$
\begin{aligned}
2 \eta^{2} \frac{\partial a}{d z}=2 g(z) \frac{2 \eta^{2} a^{2}}{a}+2 \epsilon \eta^{2} a^{2}\left(\frac{2 a}{3}+\omega^{2} \frac{2}{q}\right), & \\
4 i \eta^{2}\left(\omega \frac{\partial a}{\partial z}+a \frac{\partial a}{\partial z}\right)= & 2 g(z) \cdot 4 \eta^{2} a \cdot i \omega \\
& +2 \epsilon 4 \eta^{2} a i \omega \cdot\left(a^{2}+\omega^{2}\right) .
\end{aligned}
$$

By insertion of (A.4a) in (A.4b) we find an expression for the change in signal frequency with respect to the propagating distance $z$ as a function of the gain curvature:

$$
\frac{\partial \omega}{\partial z}=\frac{4}{3} \epsilon \omega a^{2}
$$

For a flat gain curve, $\epsilon=0$ and the optical signal frequency does not change. A typical gain shape results in a curvature leading to $\epsilon<0$. Thus, if the angular frequency $\omega_{0}$ is reduced relative to the initial optical signal frequency, then $\partial \omega / \partial z>0$. Similarly if $\omega_{0}$ is increased, $\partial \omega / \partial z<0$. This implies the guiding property of the gain curvature. In [16] a perturbation to the soliton spectra is given by

$$
\frac{\partial \omega}{\partial z}=\frac{4}{3} \epsilon a^{2} \omega+\psi(z),
$$

where the mean value $\langle\psi(z)\rangle=0$ and the two-point correction $\left\langle\psi(z) \cdot \psi\left(z^{\prime}\right)\right\rangle=C_{0} \delta\left(z-z^{\prime}\right)$. Introducing $\alpha=\frac{4}{3} \cdot \epsilon a^{2}$, a solution to (A.6) is

$$
\omega(z)=e^{\alpha z}\left(\int_{0}^{z} e^{-\alpha s} \psi(s) d s\right)
$$

which has mean value

$$
\langle\omega(z)\rangle=e^{\alpha z}\left(\int_{0}^{z} e^{-\alpha s}\langle\psi(s)\rangle d s\right)=0
$$

and two-point correction

$$
\begin{aligned}
\left\langle\omega(z) \cdot \omega\left(z^{\prime}\right)\right\rangle=\frac{C_{0}}{2 \alpha}\left\{\exp \left(\alpha\left|z-z^{\prime}\right|\right)\right. & \\
& \left.-\exp \left(\alpha\left(z+z^{\prime}\right)\right)\right\} .
\end{aligned}
$$

A shift in the signal frequency is equivalent to a shift in the arrival time of a pulse. From (A.9) the two-point correction of the pulse arrival time is expressed as

$$
\left\langle\delta t(z) \cdot \delta t\left(z^{\prime}\right)\right\rangle=\int_{0}^{z} \int_{0}^{z^{\prime}}\left(\beta_{2}\right)^{2}\langle\omega(s) \cdot \omega(t)\rangle d s d t .
$$

When the mean value $\langle\omega(z)\rangle=0$, the variance in pulse arrival time is found through (A.10). Using the notation

$$
f_{1}(u, v)=-\frac{C_{0}}{2 \alpha}\{\exp (\alpha(u, v))-\exp (\alpha(u+v))\},
$$

$$
f_{2}(u, v)=-\frac{C_{0}}{2 \alpha}\{\exp (\alpha(u, v))-\exp (\alpha(u+v))\}
$$

(A.10) is expressed as

$$
\begin{aligned}
\left\langle\delta t(z) \cdot \delta t\left(z^{\prime}\right)\right\rangle=\left(\beta_{2}\right)^{2} & \left\{\int_{0}^{z^{\prime}} \int_{0}^{z} f_{2}(u, v) d u d v\right. \\
& \left.+\int_{0}^{z^{\prime}} \int_{0}^{v} f_{1}\left(u_{1} v\right) d u d v\right\} .
\end{aligned}
$$

The variance is then found by setting $z=z^{\prime}$.

The constant $C_{0}$ is found by using the boundary condition of $\alpha \rightarrow 0$ equivalent to the ordinary timing jitter [9]. Thus we identify $C_{0}$ as

$$
C_{0}=2 \cdot \frac{E_{\mathrm{ASE}}}{E_{\mathrm{sig}}} \cdot \omega_{\mathrm{rms}}^{2} \cdot \frac{1}{\Delta z},
$$

and the reduction in timing jitter when the gain profile is included is

$$
\frac{3}{2} \cdot \frac{3+e^{2 \alpha z}+2 \alpha z-4 e^{\alpha z}}{(\alpha z)^{3}}
$$




\section{APPENDIX B}

Derivation of THE Noise Figure in a d-EDF

The signal-to-noise ratio at the output of an amplifier with gain $G$ is

$$
\left(\frac{\mathrm{S}}{\mathrm{N}}\right)_{\text {out }}=\frac{\langle n\rangle^{2}}{v},
$$

where $\langle n\rangle$ is the mean number of signal photons $n$ and $v$ is the variance of $n$. From [24] we adopt the mean number of photons

$$
\langle n\rangle=G n_{0}+M q(G-1),
$$

where $q$ is defined by (18) in the main text, $n_{0}$ is the emitted signal photons, $M=K \cdot B_{0} \cdot \Delta T$ is the number of signal modes to which the receiver is sensitive, $B_{0}$ is the detector bandwidth, $\Delta T$ the time constant of the integrator in the detector, and $K$ equals 1 or 2 if the detector is sensitive to one or, respectively, two polarizations. Assuming a coherent signal source, the variance of the photons at the signal output end is [24]

$$
\begin{aligned}
v=G n_{0}+M q(G-1)+2 q n_{0} G & (G-1) \\
& +M q^{2}(G-1)^{2} .
\end{aligned}
$$

Thus,

$$
\left(\frac{\mathrm{S}}{\mathrm{N}}\right)_{\text {out }}=\frac{G^{2} n_{0}^{2}+2 G n_{0} M q(G-1)}{G n_{0}+M q(G-1)+\begin{array}{r}
+2 q n_{0} G(G-1) \\
+M q^{2}(G-1)^{2}
\end{array}}
$$

The noise figure is defined as $F=(\mathrm{S} / \mathrm{N})_{\text {in }} /(\mathrm{S} / \mathrm{N})_{\text {out }}$, where $(\mathrm{S} / \mathrm{N})_{\text {in }}$ is the signal-to-noise ratio at the signal input end. Assuming a coherent signal source $(\mathrm{S} / \mathrm{N})_{\text {in }}=$ $n_{0}$, therefore,

$$
F=\frac{G n_{0}^{2}+n_{0} M q(G-1)+2 q G n_{0}^{2}(G-1)}{G^{2} n_{0}^{2}+2 G n_{0} M q(G-1)+M^{2} q^{2}(G-1)^{2}} .
$$

Using the approximations $M q(G-1)+M q^{2}(G-1)^{2} \ll$ $2 q G(G-1) n_{0}$ and $G^{2} n_{0}^{2} \gg G n_{0} M q(G-1)+M^{2} q^{2}(G$ $-1)^{2}$, the noise figure reduces to

$$
F=\frac{1+2 q(G-1)}{G} .
$$

The above approximation states that the signal spontaneous beat noise exceeds the shot noise and spontaneous-spontaneous beatnoise. In linear transmission systems this is normally satisfied. However, especially for solitary transmission systems, the approximation is even closer to the true description, due to the high signal power.

Pictorially the d-EDF may be considered as a cascade coupling of an infinite number of amplifiers each with gain $G_{A}$ and noise figure $F_{A}$. The amplifiers are separated with a pssive fiber section having an attenuation $D_{B}$ that equalizes $G_{A}, D_{B}=G_{A}$. The total number of emitted ASE photons is then $n_{\mathrm{ASE}}=N \cdot\left(F_{A} \cdot G_{A}-1\right)$. Thus the total noise figure is

$$
F_{\mathrm{tot}}=2 q N\left(G_{A}-1\right)+1 .
$$

Expressing the gain $G_{A}$ through the relation

$$
\begin{aligned}
G_{A}=\sqrt[N]{D_{B}}=1+\frac{\ln D_{B}}{N}+ & \frac{1}{2 !}\left(\frac{\ln D_{B}}{N}\right)^{2} \\
& +\frac{1}{3 !}\left(\frac{\ln D_{B}}{N}\right)^{3}+\cdots,
\end{aligned}
$$

thus by insertion of (B.8) in (B.7),

$$
F_{\text {tot }}=2 q \ln D_{B}+1 \text {. }
$$

\section{ACKNOWLEDGEMENT}

The authors wish to thank Dr. E. Nicolaisen for fruitful discussions.

\section{REFERENCES}

[1] A. Hasegawa and Y. Kodama, "Signal transmission by optical solitons in monomode fiber," Proc. IEEE, vol. 89, no. 9, pp. 1145-1150, 1981.

[2] L. F. Mollenauer, M. J. Neubelt, S. G. Evangiledes, J. P. Gordon, J. R. Simpson, and L. G. Cohen, "Experimental study of soliton transmission over more than $10,000 \mathrm{~km}$ in dispersion-shifted fiber," Opt. Lett., vol. 15, no. 15, pp. 1203-1205, 1990.

[3] L. F. Mollenauer, J. P. Gordon and M. N. Islam, "Soliton propagation in long fibers with periodically compensated loss," J. Quantum Electron., vol. QE-22, no. 1, pp. 157-173, 1986.

[4] J. P. Gordon and L. F. Mollenauer, "Effects of fiber nonlinearities and amplifier spacing on ultra-long distance transmission," $J$. Lightwave Technol., vol. 9, no. 2, pp. 170-173, 1991.

[5] K. Rottwitt, A. Bjarklev, J. H. Povlsen, and T. Rasmussen, "Design of long distance distributed erbium doped fiber amplifier," Electron. Lett., vol. 28, no. 3, pp. 287-288, 1992.

[6] K. Rottwitt, J. H. Povlsen, A. Bjarklev, O. Lumholt, B. Pedersen, and T. Rasmussen, "Optimum signal wavelength for a distributed erbium-doped fiber amplifier," Photon. Tech. Lett., vol. 4, no. 7, pp. 714-717, 1992.

[7] L. F. Mollenauer, S. G. Evangiledes, H. A. Haus, "Long-distance soliton propagation using lumped amplifiers and dispersion shifted fiber," J. Lightwave Technol., vol. 9, no. 2, pp. 194-197, 1991.

[8] A. Hasegawa and Y. Kodama, "Guiding-center soliton in optical fibers," Opt. Lett., vol. 15, pp. 1443-1445, no. 24.

[9] K. Rottwitt, A. Bjarklev, J. H. Povlsen, O. Lumholt, and T. Rasmussen, "Fundamental design of a distributed erbium doped fiber amplifier for long distance transmission," J. Lightwave Technol., vol. 10, no. 11, pp. 1544-1552, 1992.

[10] B. Pedersen, A. Bjarklev, J. H. Povlsen, K. Dybdal, and C. C. Larsen, "The design of erbium-doped fiber amplifiers," J. Lightwave Technol., vol. 9, no. 9, pp. 1105-1112, 1991.

[11] S. P. Craig-Ryan, J. F. Massicott, M. Wilson, B. J. Ainslie, and R. Wyatt, "Optical study of low concentration $\mathrm{Er}^{3+}$ fibres for efficient power amplifiers," Proc. European Conference on Optical Communication, ECOC'90, 1990, vol. 1, pp. 571-574.

[12] K. Rottwitt, J. H. Povlsen, A. Bjarklev, O. Lumholt, B. Pedersen, F. B. Pedersen, and T. Rasmussen, "Detailed analysis of distributed erbium doped fibre amplifiers," Proc. Optical Amplifiers and their Application, OAA'92, 1992, paper FB6, pp. 178-181.

[13] J. R. Simpson, H-T. Shang, L. F. Mollenauer, N. A. Olsson, P. C. Becker, K. S. Kranz, P. J. Lemaire, and M. J. Neubelt, "Performance of a distributed erbium-doped dispersion-shifted fiber amplifier," J. Lightwave Technol., vol. 9, no. 2, pp. 228-233, 1991. 
[14] K. Rottwitt, J. H. Povlsen, and A. Bjarklev, "Demand for accuracy of the attenuation constant in distributed active fibers," Proc. SPIE'91 OE /FIBERS, 1991, paper 1581-20.

[15] C. Lester, K. Rottwitt, and J. H. Povlsen, "Stimulated Raman scattering in distributed erbium doped fibres," ECOC'93, paper Tu1.

[16] S. Wen and S. Chi, "Distributed erbium-doped fibre amplifiers with stimulated Raman scattering," IEEE Photon. Tech. Lett., vol. 4, no. 2, pp. 189-192, 1992

[17] D. N. Chen and E. Desurvire, "Noise performance evaluation of distributed erbium-doped fiber amplifiers with bidirectional pumping at $1.48 \mu \mathrm{m}$," IEEE Photon. Tech. Lett., vol. 4, no. 1, pp. 52-55, 1992.

[18] Y. Kodama and A. Hasegawa, "Generation of asymptotically stable optical solitons and suppression of the Gordon-Haus effect," Opt. Lett., vol. 17, no. 1, pp. 31-33, 1992.

[19] D. Marcuse, "Simulations to demonstrate reduction of the Gordon-Haus effect," Opt. Lett., vol. 17, no. 1, pp. 34-36, 1992.

[20] Y. Kodama, M. Romagnoli, and S. Wabnitz, "Soliton stability and interactions in fibre lasers," Electron. Lett., vol. 28, no. 21, pp. 1981-1983, 1992.

[21] L. F. Mollenauer, J. P. Gordon, and S. G. Evangiledes, "The sliding-frequency filter: An improved form of soliton jitter control," Opt. Lett., vol. 17, no. 22, pp. 1575-1577, 1992.

[22] N. A. Olsson, "Lightwave systems with optical amplifiers," $J$. Lightwave Technol., vol. 7, no. 7, pp. 1071-1082, 1989.

[23] L. F. Mollenauer, E. Lichtman, G. T. Harvey, M. J. Neubelt, and B. M. Nyman, "Demonstration of error-free soliton transmission over more than $15000 \mathrm{~km}$ at $5 \mathrm{Gbit} / \mathrm{s}$, single-channel, and over more than $11000 \mathrm{~km}$ at $10 \mathrm{Gbit} / \mathrm{s}$ in two-channel WDM," Electron. Lett., vol. 28, no. 8, pp. 792-794, 1992.

[24] E. Desurvire, "Spectral noise figure of $\mathrm{Er}^{3+}$-doped fiber amplifiers," IEEE Photon. Tech. Lett., vol. 2, no. 3, pp. 208-210, Mar. 1990.

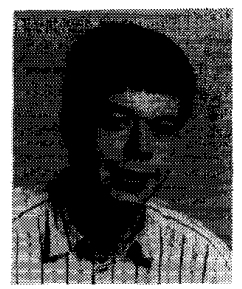

Karsten Rottwitt was born in Odense, Denmark on November 13, 1965. He received the M.Sc degree in Electrical Engineering in 1990 from the Electromagnetics Institute, Technical University of Denmark. He is presently working at the Center for Broadband Telecommunications Electromagnetics Institute on active optical waveguides and soliton transmission.

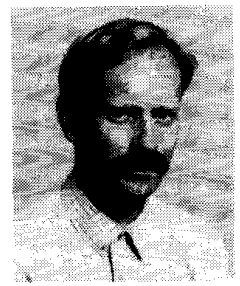

Jørn Hedegaard Povlsen received the M.Sc. degree in Physics from the H. C. Ørsted Institute of Copenhagen, Denmark, in 1982. In 1983 he joined the Electromagnetics Institute, Technical University of Denmark, as an Associate Researcher. His interests are in the fields of characterization of active and passive optical waveguides and MQW structures.

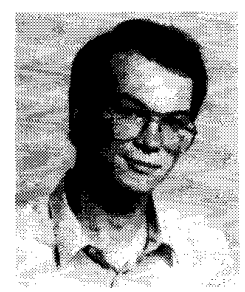

Anders Bjarklev was born in Roskilde, Denmark, on July 2, 1961. He received the M.Sc. degree in Electrical Engineering in September, 1985 from the Electromagnetics Institute, Technical University of Denmark. He received the $\mathrm{Ph} . \mathrm{D}$. degree from the Technical University of Denmark in 1988. His research interests are primarily within the field of the characterization of active and passive optical waveguides. Presently, he is employed as assistant professor versity of Denmark. at the Electromagnetics Institute, Technical uni- 